\title{
ANIMACIDADE NA SINTAXE: UMA ABORDAGEM FORMAL
}

\section{ANIMACY IN SYNTAX: A FORMAL APPROACH ${ }^{1}$}

Sonia Cyrino

Departamento de Linguística, Instituto de Estudos da Linguagem, Universidade Estadual de Campinas

Campinas, São Paulo, Brasil

\begin{abstract}
RESUMO: Uma observação recorrente no estudo da realização do objeto direto em algumas línguas românicas reside no fato de que traços de animacidade parecem determinar a forma que o objeto toma. Neste trabalho, investigo fenômenos que envolvem o traço animacidade e que foram observados separadamente na literatura sobre as línguas românicas: Marcação Diferencial do Objeto, leísmo e a Restrição Caso-Pessoa. O objetivo foi analisar esses fenômenos em comparação com o objeto nulo do português brasileiro, com a finalidade de determinar o papel que a animacidade tem na sintaxe. Os resultados da pesquisa demonstram que, de fato, a animacidade é relevante para a sintaxe. A partir desses resultados, avanço uma proposta que pretende dar conta dos fenômenos investigados e dá suporte a uma abordagem formal para as hierarquias de referencialidade encontradas na literatura não-formalista.
\end{abstract}

PALAVRAS-CHAVE: Animacidade; Hierarquia referencial; Objetos nulos; Português brasileiro.

\begin{abstract}
A recurrent observation in the study of the realization of direct objects in some Romance languages lies in the fact that animacy features seem to determine the form the object takes. In this paper, I investigate phenomena that involve animacy features and that were observed separately in the literature on Romance langauges: Differential Object Marking, leismo, and the Person-Case Constraint. The goal was to analyze these phenomena in comparison to the null object in Brazilian Portuguese, with the aim of determining the role that
\end{abstract}

\footnotetext{
${ }^{1}$ Este trabalho foi apoiado pelos seguintes projetos: CNPq 303742/2013; FAPESP 2014/17477-7 e 2012/06078-9. Gostaria de agradecer os comentários recebidos no Encontro do GT de Teoria da Gramática, UFBA, Salvador, julho 2017 e as valiosas sugestões de dois pareceristas anônimos.
} 
animacy has in syntax. The results of the research show that, in fact, animacy is relevant for syntax. Based on those results, I advance a proposal that intends to account for the phenomena and supports a formal approach to the referentiality hierarchies found in the non-formalist literature.

KEYWORDS: Animacy; Referential hierarchy; Null objects; Brazilian Portuguese. 


\section{INTRODUÇÃO}

Um tema interessante no estudo da realização do objeto em algumas línguas românicas é a referência a traços de animacidade, na medida em que são determinantes para a forma que o objeto toma: um sintagma nominal, um pronome pleno, um clítico, um objeto nulo. Por exemplo, em espanhol, francês e português europeu, pronomes objeto plenos sempre têm o traço [+animado] e são marcados pela preposição $a$, clíticos podem ser ou não animados e sintagmas nominais, se animados, são marcados pela preposição $a$.

Objetos nulos, isto é, objetos diretos que estão presentes na estrutura argumental do verbo, mas não estão presentes de uma forma audível na sentença, ocorrem no português brasileiro (doravante, $\mathrm{PB}$ ):

(1) Eu usei o vestido novo logo depois que eu comprei Ø.

Uma das peculiaridades do português brasileiro (doravante, PB) comparado com o português europeu e outras línguas que permitem a ausência do objeto direto, é que a língua permite objeto nulos anafóricos quando o antecedente é [-animado] (DUARTE, 1986; CYRINO, 1994, entre outros):

a. A Maria pegou o livro depois que o Pedro deixou Ø na mesa.

b. *A Maria beijou o Pedro depois que a Jane deixou Ø no cinema.

Porém, o trabalho de Schwenter (2006) apontou para uma co-relação entre a ocorrência de objetos nulos no PB e a Marcação Diferencial do Objeto do espanhol, pois ambos os fenômenos são sensíveis a traços como [animacidade] e [especificidade]. Essa observação trouxe elementos novos para a análise do fenômeno no PB. Este trabalho focaliza a relevância do traço [animacidade] para a sintaxe e propõe uma análise formal.

O trabalho está organizado da seguinte forma: primeiramente, apresento os fenômenos estudados na literatura e sua relação com a animacidade do objeto direto; em seguida, apresento algunas visões formais avançadas para as hierarquias de referencialidade e, finalmente, minha proposta para dar conta dos fenômenos elencados no trabalho.

\section{O TRAÇO [ANIMACIDADE] NOS FENÔMENOS SINTÁTICOS}

Como apontado acima, o traço [animacidade] pode ter uma contribuição em relação à realização dos objetos diretos em diversas línguas. Nesta seção, apresento diferentes fenômenos sintáticos que demonstram sensibilidade a esse traço: a Marcação Diferencial do Objeto (DOM, Differential Object Marking), o leísmo e a Restrição Caso-Pessoa (PCC, Person-Case Constraint), e o objeto nulo do PB. 


\subsection{A marcação diferencial do objeto}

É bem sabido que o espanhol apresenta uma marcação especial para certos objetos da língua, a chamada Marcação Diferencial de Objeto (DOM), fenômeno presente também no romeno, no hindi, no persa, e em outras línguas, como observado na literatura (AISSEN, 2003, 1991, entre outros).

De um modo geral, de acordo com a literatura, a DOM envolve: (I) propriedades morfológicas, ou seja, alguma marcação aberta; (II) propriedade sintáticas, ou seja, a posição do objeto marcado; (III) propriedade semânticas, ou seja, traços como [definitude/especificidade] e [animacidade].

Bossong (1985) afirma que "propriedades inerentes" (a animacidade do objeto) e traços referenciais (a definitude/especificidade) do objeto são importantes para a ocorrência dessa marcação. A DOM em espanhol é um caso representativo de um fenômeno mais geral nas línguas (AISSEN, 2003). Nessa língua, o objeto direto é marcado com $a$ quando é animado (ou específico, ver RODRIGUEZ-MONDOÑEDO, 2007 para uma revisão da literatura):
a. He visto *(a) tu padre. 'Vi teu pai.'
b. He visto (*a) tu coche. 'Vi teu carro.'

Vários estudos recentes têm tratado a DOM dentro de um enfoque sintático. Nesse sentido, seria o resultado do movimento do DP objeto para uma posição externa a VP, motivada por questões de Caso. Entre os autores que advogam essa visão estão Torrego (1998), Rodríguez-Mondoñedo (2007), López (2012), Ormazabal e Romero (2013) e Zrodjewski (2013), entre outros. Os três primeiros têm em comum o fato de que associam a DOM a uma configuração especial, mas cada autor apresenta uma proposta diferente para essa configuração, sendo que divergem em relação à posição para a qual o DP objeto marcado se move e à natureza dessa projeção.

Para Torrego (1998), o objeto DOM está em um segundo especificador de uma projeção $v \mathrm{P}$, que introduz o argumento externo (EA). Rodríguez-Mondoñedo (2007) não toma uma posição explícita em relação ao argumento externo, mas López (2012) argumenta que os objetos DOM estão mais baixos do que os argumentos externos, movendo-se para um núcleo intermediário entre $v \mathrm{P}$ e VP. Nesse sentido, o contraste em (4) é explicado pelo fato de o DOM não ccomandar o argumento externo:
a. *Ayer no atacó su $\mathrm{su}_{\mathrm{i}}$ propio padre a ningún $n_{\mathrm{i}}$ niño. (pretendido: Ontem seu próprio pai não atacou nenhum filho)
b. Ayer no atacó ningún $n_{i}$ padre a su $u_{i}$ propio niño. 'Ontem nenhum pai atacou seu próprio filho.'

Em (4a), o pronome possessivo não pode ter uma leitura ligada, desencadeada pela negação dentro do objeto direto. López assume que sujeitos 
pós-verbais ficam in situ no espanhol. Já em (4b), o argumento externo ccomanda o objeto DOM; portanto, a leitura ligada é possível.

Quanto à natureza da projeção para a qual o objeto DOM se move, Torrego propõe que a marca $a$ é ela própria um núcleo que traz propriedades nominais. Já para Rodríguez-Mondoñedo, a marca não está presente na sintaxe e simplesmente reflete Caso no nível morfofonológico. López assume que a marca está em um núcleo específico, K, que seleciona o objeto direto, mas regras de Spell Out serão responsáveis pela pronúncia de $a$ ou não. Assim, para o autor, $a$ é, na realidade, uma das possíveis opções para a pronúncia do núcleo K que domina o DP.

Além dessas, há outras propostas para explicar a DOM, mas estão baseadas em pressupostos um tanto diferentes: Ormazabal e Romero (2013) relacionam a DOM à concordância de objeto; Ordoñez e Roca (a sair) consideram a preposição $a$ como uma sonda; e Zdrojewski (2013) separa o movimento de DOM para uma posição do tipo $\alpha \mathrm{P}$ da necessidade de o DP obter Caso.

Ormazabal e Romero (2013) investigam DOM e sua relação com clíticos objeto em variedades do espanhol. Os autores assumem que a atribuição de Caso ao objeto vem acompanhada de concordância com o objeto, e que há somente uma posição para essa concordância. A ideia é que, sendo [+animado], o DP precisa ser licenciado por concordância. Portanto, o DP deve se mover para spec, VP. Como resultado dessa relação de checagem, temos a marcação do objeto por a. DPs indefinidos e [-animado] não recebem essa marca porque se incorporam (Ormazabal; Romero, 2013). Os autores assumem que o clítico dativo le é uma marca de concordância de objeto em $v$. Assim, quando há um objeto direto DOM (com a marca $a$ de concordância), este compete com o clítico pela mesma posição. Nesse sentido, (5a) é agramatical em contraste a (5b), em que não há a marca $a$ e, portanto, o objeto não se moveu:

a. *Le enviaron a todos los heridos a la doctora.

b. Le enviaron todos los heridos a la doctora.

'Enviaram todos os feridos à doutora.'

Ordoñez e Roca (a sair) também assumem que a DOM envolve uma projeção funcional extra. Essa projeção seria responsável por uma relação de checagem que certos objetos necessitam. Para os autores, que seguem a proposta de "preposições como sondas" à la Kayne (2005), $a$ é uma preposição inserida na derivação:

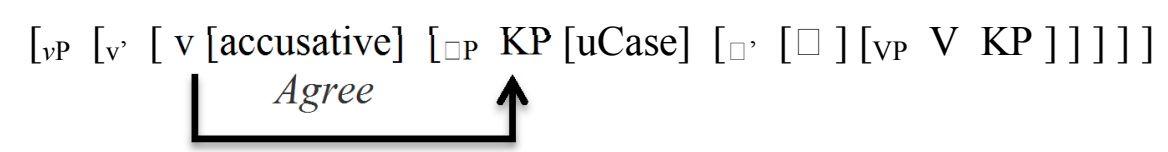

Para os autores, os objetos DOM não são licenciados por $v$ em espanhol, pois $v$ não licencia DPs objeto que portem os traços [+animado, + específico] nessa língua (ao contrário do que ocorre com $v$ em inglês). Uma vez que isso acontece, o espanhol necessita de um mecanismo extra de licenciamento: a preposição $a$ está presente na numeração e é a única licenciadora dessas construções. 
Mais recentemente, Zdrojewski (2013) apresenta uma proposta para DOM semelhante à de López (2012). Para Zdrojewski o objeto DOM também está em uma posição de especificador abaixo de $\nu \mathrm{P}$. Mas a diferença entre as propostas é que, para Zdrojewski, todos os objetos DOM ocupam a mesma posição, ao passo que os objetos não-DOM são complementos da raiz, $\sqrt{ }$.

Para o autor, a posição de DOM é uma posição que não tem traços-phi e, portanto, é uma posição (denominada $\alpha$, pelo autor) à qual Caso não pode ser atribuído; assim, o movimento do DP para o especificador de $\alpha \mathrm{P}$ não é devido a Caso. Como consequência, DOM é necessária, e atua como uma regra póssintática que satisfaz o Caso do DP. Isso é feito através de um morfema dissociado, que introduz uma projeção, KP. Pós sintaticamente, o DP em spec, $\alpha \mathrm{P}$ será complexo, lembrando o DP de López (2012).

Dessa forma, vimos que as propostas recentes para explicar sintaticamente a DOM propõe que o DP [+animado], ou seja, aquele que será marcado, seja movido para fora de VP. As propostas não apresentam argumentos empíricos para o movimento, mas assumirei essa ideia geral, como veremos abaixo.

\subsection{0 leísmo}

Denomina-se leísmo à extensão do clítico le, que seria a forma do dativo, para contextos em que se esperaria o acusativo lo ou la. Em um estudo importante sobre o fenômeno, Ormazabal e Romero (2013) mostram que essa co-relação não pode ser feita, pois a referida extensão não se concretiza em certos dialetos (espanhol centro-europeu). O interessante desses dialetos é que objetos diretos masculinos podem ser marcados por $a$ e pronominalizados por le. Porém, objetos diretos femininos e [+animado], embora possam ser marcados por $a$, não podem ser pronominalizados por le:

a. Vi al chico.

'Vi o menino.'

b. Le vi.

'Lhe vi.'
a. Vi a la chica.
'Vi a menina.'
b. $L a$ vi. ( cf. * $L e$ vi.)

O mesmo não ocorre no espanhol do país basco, em que todos os objetos diretos [+animado] são pronominalizados por $l e$ :
a. Vi el libro.
'Vi o livro.'
b. Lo vi.
'O vi.' 
a. Vi a $\{1$ chico / la chica $\}$

'Vi o menino/a menina.'

b. Le vi.

'Lhe vi.'

Esse fato demonstra que os objetos DOM, ou seja, aqueles portanto o traço [+animado], parecem ser dativos neste último dialeto. Porém, veremos abaixo como o fenômeno tem sido analisado dentro da Restrição Caso-Pessoa.

\subsection{A restrição caso-pessoa}

A Restrição Pessoa-Caso (PCC, ver Bonet, 1991, entre outros) é fenômeno no qual o objeto direto deve ser de $3^{\mathrm{a}}$. pessoa em uma estrutura bitransitiva em que ambos os argumentos são realizados como clíticos. Em outras palavras, a presença de um clítico dativo de $3^{\mathrm{a}}$. pessoa bloqueia a ocorrência de um clítico acusativo de $1^{\mathrm{a}}$. ou $2^{\mathrm{a}}$. pessoa. Em $(11 \mathrm{~b})$, por exemplo, o clítico acusativo de $2^{\mathrm{a}}$. pessoa co-ocorre com o clítico dativo de $3^{\mathrm{a}}$. pessoa, e a sentença é agramatical.

a. Os enviaron a la doctora.

'Enviaram vocês à doutora.'

$\begin{array}{lll}\text { b. }{ }^{*} \text { Le } & \text { os } & \text { enviaron. } \\ 3 \text { cl.DATIVO } & \text { 2cl.ACUSATIVO } & \text { enviaram }\end{array}$

Muitos pesquisadores explicam a PCC em termos de pessoa $\left(1^{\mathrm{a}} / 2^{\mathrm{a}}\right.$ vs $\left.3^{\mathrm{a}}\right)$, Caso (Acusativo vs. Dativo), ou em termos da natureza morfológica dos clíticos.

Ormazabal e Romero (2007), no entanto, propõem que o traço [animacidade] tem um papel determinante para o fenômeno. Os autores propõem que o clítico le é, na realidade, um marcador de animacidade. Como vimos acima, em dialetos leístas, le é usado não somente para argumentos dativos mas também para argumentos acusativos animados. Isso é claro quando se observa como esses dialetos diferenciam os clíticos acusativos por gênero e animacidade.

Embora homófono com o dativo, os autores afirmam nos dialetos leístas que há um clítico de $3^{\mathrm{a}}$. pessoa le acusativo, assim como há pronomes de $1^{\mathrm{a}}$. e $2^{\mathrm{a}}$. pessoas (me e te) acusativos e me e te dativos. Esse le somente se diferencia do pronome de $3^{\mathrm{a}}$. pessoa acusativo lo no valor para animacidade.

Nesses dialetos o clítico objeto animado le nunca pode ocorrer (em um grupo clítico) com um clítico dativo. Assim, os exemplos abaixo (exemplos (15) e (16) em ORMAZABAL; ROMERO, 2007, p. 321) mostram que quando o clítico acusativo ocorre isoladamente deve ser realizado como lo se o objeto não é animado (12a) e como le se for animado (12b). O interessante é que a PCC somente ocorre quando o clítico é o animado le (13b), mas não quando é lo (13a):
a. Lo vi. (lo $=3^{\mathrm{a}}$. pessoa acusativo [-animado])
b. $L e$ vi. $\left(l e=3^{\mathrm{a}}\right.$. pessoa acusativo [+animado]) 
(13)

a. Te lo di.

$\left(t e=2^{\mathrm{a}}\right.$. pessoa dativo $([+$ animado $]) ; l o=3^{\mathrm{a}}$. pessoa acusativo $[$-animado $\left.]\right)$

b. ${ }^{*}$ Te le di.

$\left(t e=2^{\mathrm{a}}\right.$. pessoa dativo $([+$ animado $]) ; l e=3^{\mathrm{a}}$. pessoa acusativo $[+$ animado $\left.]\right)$

Ormazabal e Romero propõem que a definição de PCC seja formulada em termos de animacidade em vez de [pessoa]. Os autores tratam a impossibilidade de haver dois DPs marcados com $a$ como sendo o efeito da competição de dois constituintes pela mesma posição de concordância do objeto.

Em seus trabalhos, Ormazabal e Romero unificam o fenômeno de DOM e a PCC. Essa unificação é possível pois os autores assumem que clíticos acusativos de $1^{\mathrm{a}}$. e $2^{\mathrm{a}}$. pessoas são, assim como os clíticos dativos, marcadores de concordância verbo-objeto, o que se pode depreender da possibilidade de reduplicação desses clíticos:

(14) a. Os veré a los que vaias pronto. ( $o s=2^{\mathrm{a}}$. pessoa plural acusativo)

b. Os veré a algunos en el examen. (os $=2^{\mathrm{a}}$. pessoa plural acusativo)

a. Les compré un libro a los primos. (les $=3^{\mathrm{a}}$. pessoa plural dativo)

b Les pagamos las deudas a algunos acreedores. (les $=3^{\mathrm{a}}$. pessoa plural dativo)

Em contraste com esses clíticos, estão os clíticos de $3^{\mathrm{a}}$. pessoa acusativo $(l o, l a)$, que os autores assumem ser um determinante, seguindo ampla literatura (ORMAZABAL; ROMERO, 2006; URIAGEREKA, 1995; ROCA, 1992, 1996). A evidência estaria no fato de que clíticos acusativos de $3^{\mathrm{a}}$. pessoa, por serem determinantes, não podem ser reduplicados (16), ao contrário dos clíticos de $1^{\mathrm{a}}$. e $2^{\mathrm{a}}$. pessoas e $3^{\mathrm{a}}$. pessoa dativos (14)-(15).

(16) a. *La vimos la casa. (la $=3^{\mathrm{a}}$. pessoa singular acusativo)

b. *Los vimos a algunos. (los $=3^{\mathrm{a}}$. pessoa plural acusativo)

Para entender a unificação proposta para PCC e DOM pelos autores, tomemos os exemplos em (11) repetidos aqui em (17). Em (17a) há somente uma marca de concordância verbo-objeto (os autores propõem que há somente uma posição no VP para essa marca): o clítico acusativo de $2^{\mathrm{a}}$. pessoa. A sentença, portanto, é gramatical. Já em (17b), temos duas potenciais marcas de concordância, pois ambos o clítico acusativo de $2^{\mathrm{a}}$. pessoa $(o s)$ e o clítico dativo de $3^{\mathrm{a}}$. pessoa (le) são, segundo os autores, marcas de concordância com o verbo. Em outras palavras, temos aí dois clíticos marcando a concordância verbo-objeto, um dativo ( $3^{\mathrm{a}}$. pessoa) e um acusativo ( $2^{\mathrm{a}}$. pessoa), ou seja, dois constituintes competindo pela mesma posição de concordância do objeto, o que provoca a agramaticalidade de (17b):

(17) a. Os enviaron a la doctora.

b. *Le os enviaron.

Assim, tanto DOM quanto a PCC são explicadas pela presença do traço [animacidade]. Como vimos acima, Ormazabal e Romero (2013) assumem que a 
atribuição de Caso ao objeto vem acompanhada de concordância com o objeto e que há somente uma posição para essa concordância. Na DOM, sendo [+animado], um DP deve se mover para spec, VP e receber Caso (associado à concordância).

\subsection{0 objeto nulo no PB}

Os objetos nulos anafóricos do PB têm um conjunto específico de propriedades. Além de haver uma restrição em relação a antecedentes animados, o objeto nulo do PB também permite leituras estrita e imprecisa (strict/sloppy readings) (CYRINO, 1994, 1997, e CYRINO; LOPES, 2016). O objeto nulo em (18) tem essas duas leituras; a sentença é, portanto, ambígua. Na leitura estrita do objeto nulo, o amigo do Pedro gastou o dinheiro do Pedro; na leitura imprecisa, no entanto, o amigo do Pedro gastou seu próprio dinheiro:

O Pedro escondeu o dinheiro no armário, mas a Maria gastou Ø na feira.

Como mostrado em Cyrino (1994, 1997), um pronome pleno, como ele em (19), somente permite a leitura estrita. Assim, a sentença em (19) só pode significar que Pedro preferiu fechar a janela de João (leitura do pronome é estrita):

O Pedro escondeu o dinheiro no armário, mas a Maria gastou ele na feira.

Cyrino $(1994,1997)$ propôs que os objetos nulos do PB são casos de elipse de DP, isto é, DPs inaudíveis que são idênticos aos seus antecedentes. A elipse é um fenômeno licenciado por uma categoria funcional; no inglês, por exemplo, a elipse de $v$ P é licenciada por V em T (LOBECK, 1995).

Desenvolvendo as propostas em Cyrino e Matos (2002, 2005), Cyrino (2013) mostra que o PB perdeu o movimento "longo" do verbo. Disso conclui que a elipse de $v \mathrm{P}$ e os objetos nulos do PB (elipse de DP) são licenciados pelo verbo em uma projeção aspectual mais baixa do que T. Desenvolvendo essa ideia, Cyrino (2016) propõe que o objeto nulo do PB é licenciado por V em AspInner, um núcleo aspectual entre $v$ P e VP (MACDONALD, 2008):

(20) objeto nulo:

a. A Ni tem lido o livro para as crianças e o Gui tem também lido para as mães

b. $[$ ср ... o livro para as crianças...] ... o Gui [T+VAux tem] [VPaux $<$ tem $>$ [AdvP [Adv também] [vP [AspInn [AspInn+v lido [vp $<$ lido $>$ [ڤp- o livro] para as mães]]]

A estrutura em (20b), correspondente à sentença em (20a), mostra que o verbo se moveu para a projeção funcional de aspecto interno, Aspect Inner (AspInn), podendo licenciar a elipse do DP o livro (para os diferentes efeitos decorrentes desse movimento, ver CYRINO, 2013). 
Mas se os objetos nulos do PB são elipse, ainda temos que explicar por que são inanimados. Além disso, objetos nulos animados são possíveis, mas são restritos: o antecedente deve ser um plural nu, como em (21):

(21) a. Os tiras insultavam $[\text { presos }]_{\mathrm{i}}$ e depois prendiam $\varnothing_{\mathrm{i}} / *^{*} \mathrm{eles}_{\mathrm{i}}$

b. Os tiras insultavam [os presos $]_{\mathrm{i}}$ e depois prendiam $* \emptyset_{\mathrm{i}} / \mathrm{eles}_{\mathrm{i}}$

Se objetos nulos são elipse de DP licenciada pelo V lexical em AspInner, e os objetos nulos são somente possíveis quando o antecedente é [-animado] ou um plural nu, a impossibilidade de ocorrência de certos objetos nulos deve estar ligada ao fato de que a elipse de DP não é licenciada nesses casos. Na seção 3, apresento uma proposta para dar conta desses fatos. Antes, apresento algumas visões formais para as hierarquias de referencialidade.

\section{VISÕES FORMAIS PARA AS HIERARQUIAS DE REFERENCIALIDADE}

Vimos que a animacidade é relevante para muitos fenômenos (morfos)sintáticos, como DOM, leísmo, PCC. Um modo de dar conta desses fenômenos é usar as hierarquias de referencilidade, como aquelas propostas em estudos tipológicos e funcionalistas (por exemplo, Silverstein 1976, entre outros). Dentro de um enfoque mais formalista, Cyrino, Duarte e Kato (2000) propuseram uma Hierarquia de Referencialidade para explicar o objeto e o sujeito nulos do PB em relação à aquisição da linguagem.

Porém, como mostrado em Carnie (2005), Merchant (2006), o poder explanatório de tais hierarquias é duvidoso dentro de uma perspectiva formal, pois não está claro qual seu papel dentro do quadro teórico. Seriam efeitos da Gramática Universal, ou derivariam de princípios cognitivos independentes?

Dentro do quadro teórico da teoria gerativa, algumas propostas surgiram para tratar as hierarquias dentro da sintaxe. Entre elas, Carnie (2005) assume Chomsky (2001) e propõe que as fases consistem de um argumento, o predicado que o introduz, e um operador temporal e cada fase corresponderia a um mapeamento entre a estrutura sintática e a semântica. Os efeitos sintáticos das hierarquias seriam o resultado desse mapeamento.

Merchant (2006) também assume que a ordem das palavras corresponde às hierarquias propostas na literatura funcionalista, e apresenta uma implementação específica, baseada em Carnie (2005). Preocupado em solucionar situações de atribuição múltipla de Caso em algumas línguas, Merchant avança a idéia de que a arquitetura funcional da sentença inclui um conjunto de núcleos funcionais para cada sujeito ou objeto. O propósito dessa hierarquia é receber, em seus especificadores, DPs que tenham variadas propriedades de proeminência que normalmente são codificadas nas hierarquias. Na proposta de Merchant, esse conjunto de projeções funcionais c-comandariam o $v \mathrm{P}$ no qual o sujeito se origina. 
Levando-se em conta que há propostas na literatura em que as hierarquias de referencialidade podem ser entendidas como codificadas na estrutura da sentença, propus, em um primeiro trabalho (CYRINO, 2008, 2009, 2012), diferentes posições para os argumentos DP de acordo com seus papéis temáticos. Para tanto, assumi PLATZACK $(2003,2008)$, autor que propõe a distribuição dos DPs em três famílias, de acordo com a posição em que são concatenados: complementos de $\mathrm{V}$, especificadores de $v$ ou especificadores de $v \mathrm{P}$.

DPs portando os papéis temáticos de Caminho (Path) e Tema (Theme) são concatenados como complementos de V. Em Cyrino (2012) proponho que no PB, esses seriam os objetos nulos no PB. Por outro lado, os objetos que normalmente são marcados com acusativo em línguas em que a DOM está operativa, são aqueles que são concatenados em spec-VP. Nessa posição, não poderíamos ter objetos nulos, pois não haveria um paralelismo exigido pela operação de elipse entre a estrutura do antecedente (este estaria mais alto) e a do objeto nulo (que estaria mais baixo).

No entanto, essa proposta é insuficiente para dar conta do comportamento dos objetos diretos em relação à animacidade: argumentos Tema não estão restritos a DPs inanimados.

Na próxima seção, apresento uma proposta que traduz a intuição presente nos trabalhos e nas propostas formais para hierarquias de referencialidade apresentados acima: o DP [+animado] deve estar estruturalmente acima do [animado].

\section{PROPOSTA: O DP [+ANIMADO] SE MOVE PARA FORA DE VP}

Proponho que a hierarquia da animacidade está codificada na geometria da árvore e que há uma categoria funcional relacionada ao traço [animacidade]. Assim, a ordem linear [animado] $>$ [inanimado] apresentada nas hierarquias referenciais representa a disposição na estrutura arbórea: o DP mais à esquerda está mais acima na árvore.

Seguindo as ideias em Ordoñez e Roca (a sair) e a intuição de RodriguezMondoñedo (2007) de que DOM está relacionado à animacidade, proponho que há um núcleo funcional $\mathrm{F}_{\text {[Pessoa] }}$ localizado abaixo de $v \mathrm{P}$ e acima de $\mathrm{V}$. $\mathrm{O}$ traço [pessoa] desse núcleo funcional possui traços EPP que atraem os DPs animados. Os DPs inanimados permanecem in situ.

Além disso, sigo Richards (2008) e proponho que o traço $[ \pm$ Pessoa] está codificado nos DPs. O Quadro 1, que resume essa proposta, mostra como traços de animacidade podem ser subsumidos no traço [Pessoa] (ver RICHARDS, 2008): 
Quadro 1: [Pessoa] e [animacidade]

\begin{tabular}{|c|c|}
\hline $1^{\mathrm{a}} \cdot / 2^{\mathrm{a}} \cdot$ pessoa & {$[+$ Pessoa] } \\
\hline $3^{\mathrm{a}} \cdot$ pessoa [animado] & {$[-$ Pessoa $]$} \\
\hline $3^{\mathrm{a}} \cdot$ pessoa [inanimado]/ Plural $\mathrm{Nu}$ & 'Person-less' \\
\hline
\end{tabular}

Fonte: Cyrino 2016

Em relação à estrutura, assumo que, assim como no espanhol (RODRIGUEZ-MONDOÑEDO, 2007; ORDOÑEZ; ROCA, a sair), vs transitivos no $\mathrm{PB}$ são $\varphi$-incompletos. O Caso acusativo é somente valorado para DPs que têm os mesmos traços; dessa forma, vs não podem valorar o Caso em DPs animados, pois estes são $\varphi$-completos. Os efeitos que vemos na sintaxe em relação à animacidade são o resultado do movimento de um DP [+Pessoa] ou [Pessoa] (isto é, um DP especificado para Pessoa) para o especificador de uma categoria funcional $\mathrm{F}_{\text {[Pessoa] }}$ localizada abaixo de $v \mathrm{P}$ e acima de AspInn. Essa categoria tem o traço não-interpretável [uPessoa] e um traço EPP.

DPs [-animado] são DPs que não têm Pessoa, são 'Person-less' (ver também RICHARDS, 2008) e, de acordo com a presente análise, não se movem para fora de VP. Sendo $\varphi$-incompletos, têm o Caso Acusativo valorado pela sonda $v$ que é também $\varphi$-incompleta (como no sistema proposto por RODRIGUEZMONDOÑEDO, 2007).

Porém, DPs [+animado] são DPs que têm traços [+Pessoa] ou [-Pessoa]. O traço EPP da categoria funcional $\mathrm{F}_{\text {[Pessoa] }}$ desencadeia o movimento desses DPs para seu especificador. Nessa posição terão Caso; o movimento em si, no entanto, não é desencadeado por essa razão.

Com essa proposta podemos explicar o padrão encontrado na ocorrência do objeto nulo no PB. Vejamos as derivações para explicá-lo:

(A) DPS INANIMADOS E PLURAIS NUS:

Observe o contraste nas sentenças abaixo:

(22) a. O Pedro trouxe o livro para a escola e o professor examinou Ø.

b. *O Pedro trouxe o amigo para a escola e o professor examinou Ø.

(23) a. Os tiras insultavam [presos $]_{\mathrm{i}}$ e depois prendiam $\varnothing_{\mathrm{i}} / *^{*}$ eles $_{\mathrm{i}}$

b. Os tiras insultavam [os presos $]_{\mathrm{i}}$ e depois prendiam $* \varnothing_{\mathrm{i}} /$ eles $_{\mathrm{i}}$

(24) Os tiras puxavam $[\text { armas }]_{\mathrm{i}}$ e depois escondiam $\emptyset_{\mathrm{i}} / *^{*}$ elas $_{\mathrm{i}}$

Os DPs inanimados (22a) e os plurais nus ((23a) e (24)) são 'Person-less' e, portanto, permanecen in situ. Permanecendo in situ, o objeto nulo pode ser licenciado pelo V em AspInn:

$$
\begin{aligned}
& \text { [vP O professor } v \text { [AspInnP [ } \mathrm{v}+\text { Asp } \text { examinou [vp }<\mathrm{V}>\quad \text { o livro }]]] \\
& \text { licenciamento da elipse de DP }
\end{aligned}
$$


Esses DPs (inanimados e plurais nus) não são marcados diferencialmente em espanhol, conforme a literatura (ver Fábregas 2013).

(B) DPS ANIMADOS

Os DPs animados ( $3^{\mathrm{a}}$. pessoa) são [- Pessoa] e, portanto, se movem para

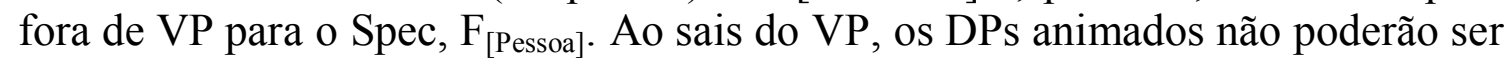
licenciados pois estarão fora do domínio de c-comando do $\mathrm{V}$ em AspInn, o licenciador de elipse do objeto. Nesse caso, um pronome anafórico pleno elelela será possível (26), com a estrutura em (27):

(26) a. O Pedro trouxe o amigo para a escola e o professor examinou ele.

b. *O Pedro trouxe o amigo para a escola e o professor examinou $\varnothing$.

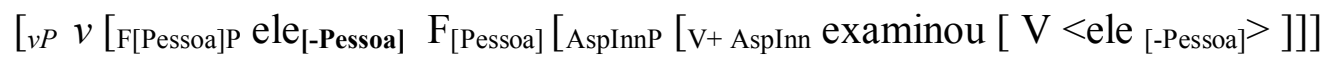

Note que um pronome pleno como ela em PB também pode se referir a um antecedente inanimado (28a). Note, porém, que o antecedente do pronome pleno precisa ser específico, dado o contraste visto em (28b), em que o antecedente é [específico]:

(28) a. O tira usa [aquela arma de fogo $]_{\mathrm{i}}$ e depois esconde $\varnothing_{\mathrm{i}} / \mathrm{ela}_{\mathrm{i}}$ para não ser preso.

b. O tira usa $[\operatorname{arma}(\mathrm{s}) \text { de fogo }]_{\mathrm{i}}$ e depois esconde $\emptyset_{\mathrm{i}} / \#$ ela $\mathrm{a}_{\mathrm{i}}$ para não ser preso.

Diferentemente de Rodriguez-Mondoñedo (2007), assumo que especificidade é um traço independente de animacidade e deriva de outros princípios na interface com a semântica. Da mesma forma, Leonetti (2008) considera que a especificidade não é um traço semântico que nós sintáticos possam codificar; a especificidade é, na verdade, obtida no processo de recuperação das proposições explicitamente comunicadas na fala (ver também LÓPEZ, 2012 e IRIMIA; CYRINO, 2015, 2017).

Minha proposta pode ser estendida para objetos DOM do espanhol, uma vez que a literatura propõe que estejam fora de VP, conforme visto acima. Da mesma forma, podemos estender a proposta para explicar a PCC. Vimos que clítico le do espanhol é um marcador de animacidade (ORMAZABAL; ROMERO, 2007). A PCC seria, portanto, decorrente do fato de haver somente uma posição para nominais animados, que estariam estruturalmente acima de inanimados.

\section{CONCLUSÃO}

Os resultados deste trabalho levam à conclusão de que as hierarquias relacionais e semânticas apresentadas na literatura funcionalista podem ser mapeadas a partir da estrutura sintática. Se isso for verdade, DPs animados estão estruturalmente posicionados acima de inanimados, e esse fato pode explicar os fenômenos em que a animacidade é relevante para a sintaxe. Acredito que esse é 
um caminho de pesquisa que poderá ser explorado em futuros estudos abrangendo outras línguas e outros fenômenos que tornem evidente a relevância sintática de traços referenciais.

\section{REFERÊNCIAS}

AISSEN, J. Differential Object marking: Iconicity vs. Economy. Natural Language and Linguistic Theory, Dordrecht, n. 21, 2003, p. 435-483.

BONET, E. Morphology after syntax: pronominal clitics in Romance. Cambridge, Massachusetts: 1991. 250 f. Tese (Doutorado em Linguística), Massachusetts Institute of Technology, Cambridge.

BOSSONG, G. Differentielle Objektmarkierung in den Neuiranisdchen Sprachen. Tübingen: Gunter Narr Verlag. 1985.

CARNIE, A. A phase-geometric approach to multiple marking systems. In: MCGINNIS, M.; RICHARDS, N. (Org.) Perspectives on phases. MIT Working Papers in Linguistics, Cambridge, Massachusetts, n. 49, 2005, p. 87-102.

CHOMSKY, N. Derivation by phase. In: KENSTOWICZ, M. (Org.). Ken Hale: a life in language. Cambridge, Massachusetts: MIT Press, 2001. p. 1-52.

CYRINO, S. O objeto nulo no português brasileiro: um estudo sintáticodiacrônico. Campinas, SP: 1994. 217 f. Tese (Doutorado em Linguística), Universidade Estadual de Campinas, Campinas.

CYRINO, S. O objeto nulo no português brasileiro: um estudo sintáticodiacrônico. Londrina: Editora da UEL. 1997.

CYRINO, S. Objetos nulos no português brasileiro e espanhol da América do Sul. Relatório final para a FAPESP, Processo no. 06/03343-2. 2008.

CYRINO, S. Null objects in New Romance: aspectuality, transitivity and referentiality. XXXI Deutscher Romanistentag, Rheinische Friedrich-WilhelmsUniversität, Bonn. Bonn, 2009.

CYRINO, S. Null objects in New Romance: Aspectuality, transitivity and referentiality. In: BELLOSTA VON COLBE, V.; GARCÍA GARCÍA, M. (Org.). Aspectualidad - Transitividad - Referencialidad: Las lenguas románicas en contraste. Frankfurt am Main: Peter Lang, 2012, p. 41-68.

CYRINO, S. On richness of tense and verb movement in Brazilian Portuguese. In: CAMACHO-TABOADA, V.; JIMÉNEZ-FERNÁNDEZ, A.; MARTÍNGONZÁLEZ, J.; REYES-TEJEDOR, M. (Org.). Information structure and agreeement. Amsterdam: John Benjamins, 2013, p. 297-317. 
CYRINO, S. Animacy and null objects in Brazilian Portuguese. Linguistics Colloquium Series, Stony Brook University. 2016.

CYRINO, S.; MATOS, G. VP elllipsis in European and Brazilian Portuguese - a comparative analysis. Journal of Portuguese Linguistics. Lisboa, v. 1, n. 2, 2002, p. 177-196.

CYRINO, S.; MATOS, G. Local licensers and recovering in VP ellipsis. Journal of Portuguese Linguistics. Lisboa v. 4, n. 2, 2005, p. 79-112.

CYRINO, S.; MATOS, Ga. Null objects and VP ellipsis. In: WETZELS, W.L.; COSTA, J.; MENUZZI, S. (Org.). The handbook of Portuguese linguistics. New York: Wiley-Blackwell, 2016. p. 294-317.

CYRINO, S.; LOPES, R. Null objects as ellipsis. Going Romance Conference, Katholieke Universiteit, Leuven, Belgium, 2012.

CYRINO, S.; LOPES, R. Null objects are ellipsis in Brazilian Portuguese. The Linguistic Review. Amsterdam, v. 33, n. 4, 2016, p. 483-502.

CYRINO, S.; DUARTE, M. E.; KATO, M. Visible subjects and invisible clitics in Brazilian Portuguese. In: KATO, M.; NEGRÃO, E. (Org.). Brazilian Portuguese and the Null Subject Parameter. Frankfurt am Main: Vervuert, 2000. p. 55-73.

CYRINO, S.; ESPINAL, M. T. Bare Nominals in Brazilian Portuguese: more on the DP/NP analysis. Natural Language \& Linguistic Theory. Dordrecht v. 33, n . 2, 2015, p. 471-521.

DUARTE, M. E. L. Variação e Sintaxe: Clítico Acusativo, Pronome Lexical e Categoria Vazia no Português do Brasil. São Paulo, SP: 1986. 150 f. Dissertação. (Mestrado em Linguística), Pontifícia Universidade Católica, São Paulo.

FÁBREGAS, A. Differential object marking in Spanish: state of the art. Borealis: An International Journal of Hispanic Linguistics. Tromso v. 2, n. 2, 2013, p. 1-80.

IRIMIA, M.-A.; CYRINO, S.. Syntactic positions for DOM: Brazilian Portuguese and Romanian. $29^{\text {th }}$ Going Romance, Radboud University, Nijmegen. 2015.

IRIMIA, M-A.; CYRINO, S. Unifying differential marking: from Brazilian Portuguese to adpositional DOM. Revue Roumaine de Linguistique. Bucarest v. LXII, n. 4, 2017, p. 411-426.

LEONETTI, M. Specificity in clitic doubling and in differential object marking. Probus. Amsterdam, v. 20, n. 1, 2008, p. 33-66.

LOBECK, A. Ellipsis: functional heads, licensing and identification. New York: Oxford University Press. 1995. 
LÓPEZ, L. Indefinite objects: scrambling, choice functions, and differential marking. Cambridge: MIT Press. 2012.

KAYNE, R. Prepositons as probes. In: KAYNE, R. (Org.). Movement and Silence. Oxford: Oxford: University Press, 2005, p. 83-104.

MACDONALD, J. The syntactic nature of inner aspect: a minimalist perspective. Amsterdam: John Benjamins. 2008.

MATOS, G. Construções de Elipse do Predicado em Português - SV Nulo e Despojamento. Lisboa. 1992. 459 f. Tese. (Doutorado em Letras), Universidade de Lisboa.

MERCHANT, J. Polyvalent case, geometric hierarchies, and split ergativity. Proceedings from the Annual Meeting of the Chicago Linguistic Society. Chicago v. 42 , n. 2, 2006, p. 57-76.

ORDOÑEZ, F.; ROCA, F. Differential Object Marking (DOM) and clitic subspecification in Catalonian Spanish. In: GÁlLEGO, A. The Syntactic Variation of Spanish Dialects. Oxford: Oxford University Press. A sair.

ORMAZABAL, J.; ROMERO, J. The object agreement constraint. Natural Language \& Linguistic Theory. Dordrecht, v. 25, n. 2, 2007, p. 315-347.

ORMAZABAL, J.; ROMERO, J. Object clitics, agreement and dialectal variation. Probus. Amsterdam v. 25, n. 2, 2013, p. $301-344$.

PLATZACK, C. Agreement and null subjects. Nordlyd. Oslo, v. 31, n. 2, 2003, p. 326-355.

PLATZACK, C. class notes. Summer Institute, GLOW, Barcelona. 2008.

RICHARDS, M. Defective agree, case alternations and the prominence of person. In: RICHARDS, M.; MALCHUKOV, A. (Org.). Scales. Linguistiche Arbeits Berichte Universität Leipzig, v. 86, 2008, p. 137-161.

ROCA, F. Object clitics in Spanish and Catalan. Catalan Working Papers in Linguistics (CWPL). Barcelona v. 2, 1992, p. 245-280.

ROCA, F. Morfemals objetivos y determinantes: los clíticos del español. Verba. Santiago de Compostela v. 23, 1996, p. 83-119.

RODRÍGUEZ-MONDOÑEDO, M. The syntax of objects: agree and Differential Object Marking. Storrs, Connecticut: 2007. 335 f. Tese (Doutorado em Linguística) University of Connecticut, Storrs.

SCHWENTER, S. Null objects across South America. Selected papers of the $8^{\text {th }}$ Hispanic Linguistics Symposium, 2006, p. 23-36. 
SILVERSTEIN, M. Hierarchy of features and ergativity. In: DIXON, R.M. (Org.). Grammatical categories in Australian languages. Atlantic Highlands: Humanities Press, 1976, p. 112-171.

TORREGO, E. The dependency of objects. Cambridge, MA: MIT Press. 1998.

URIAGEREKA, J. Aspects of the syntax of clitic placement in Western Romance. Linguistic Inquiry. Cambridge, Massachusetts, v. 26, 1995, p. 79-124.

ZDROJEWSKI, P. Spanish DOM as a case of lacking case. Universidad Nacional de General Sarmiento: Universidad de Buenos Aires. 2013.

Sonia Cyrino Cyrino@iel.unicamp.br Recebido em: 4 jan. 2018 Aceito em: 4 abr. 2018 Publicado em: 1 out. 2018 